\title{
Detecting intermediate protein conformations using algebraic topology
}

\author{
Nurit Haspel ${ }^{1 *}$, Dong Luo ${ }^{1}$ and Eduardo González ${ }^{2}$ \\ From 6th IEEE International Conference on Computational Advances in Bio and Medical Sciences (ICCABS) \\ Atlanta, GA, USA. 13-15 October 2016
}

\begin{abstract}
Background: Understanding protein structure and dynamics is essential for understanding their function. This is a challenging task due to the high complexity of the conformational landscapes of proteins and their rugged energy levels. In particular, it is important to detect highly populated regions which could correspond to intermediate structures or local minima.
\end{abstract}

Results: We present a hierarchical clustering and algebraic topology based method that detects regions of interest in protein conformational space. The method is based on several techniques. We use coarse grained protein conformational search, efficient robust dimensionality reduction and topological analysis via persistent homology as the main tools. We use two dimensionality reduction methods as well, robust Principal Component Analysis (PCA) and Isomap, to generate a reduced representation of the data while preserving most of the variance in the data.

Conclusions: Our hierarchical clustering method was able to produce compact, well separated clusters for all the tested examples.

Keywords: Algebraic topology, Protein conformational sampling, Clustering, Protein structure, Dimensionality reduction

\section{Background}

Characterizing the conformational space of proteins is crucial for understanding the way they perform their function. Understanding the connection between protein structure, dynamics and function can contribute substantially to our understanding of cellular processes involving proteins. The question of how the structure and dynamics of proteins relate to their function has challenged scientists for several decades but still remains open. Conformational exploration methods aim to characterize the conformational space of proteins in order to find minimum energy regions corresponding to highly populated structures [1,2]. These intermediate states are transient and therefore hard to detect experimentally. However, they may be crucial to understanding dynamic events such

*Correspondence: nurit.haspel@umb.edu

${ }^{1}$ Department of Computer Science, University of Massachusetts Boston, 100 Morrissey Blvd., 02125 Boston, MA, USA

Full list of author information is available at the end of the article as folding, docking, binding and conformational change processes. The potential energy landscape of a protein is often rugged and has a large number of local minima [3]. This makes it difficult to navigate. The problem becomes even more challenging due to the fact that a typical protein can contain several hundreds of amino acids or several thousands of atoms. Therefore, the search space made out of all possible conformations that a protein can assume is large and its enumeration is practically impossible. Existing physics-based computational methods that sample the conformational space of proteins include Molecular Dynamics (MD) [4], Monte Carlo (MC) [5] and their variants, as well as approximate methods based on geometric sampling [2, 6-8], Elastic Network Modeling [9, 10], normal mode analysis [11, 12], morphing [13] and several other methods.

Even after the conformational space is sampled, it should be filtered and clustered to extract meaningful information. Several clustering methods have been designed for protein conformational space $[6,14,15]$. The 
majority of clustering methods for high-dimensional data incorporates metric functions that evaluate the distance between objects in the dataset, or a lower-dimensional representation of these objects, often trying to detect outliers [16].

Hierarchical clustering methods result in a multi-scale view of the conformational space and enable us to view the hierarchical relationship between the local minima produced by the conformational search. In this work we use both algebraic topology and dimensionality reduction methods to explore and characterize the conformational space of proteins. Algebraic topology has been used in the past for clustering data [17] and exploring the conformational space of small peptides, including finding metastable states [15]. In previous work $[18,19]$ we used persistent homology to explore the conformational space of proteins and detect regions of interest that may correspond to local minima, which are hard to detect experimentally due to the relatively short time the protein spends in them. We used only standard Principal Component Analysis (PCA), whose linear nature may not capture the complex, non-linear nature of the conformational spaces. Standard PCA is also known to be highly sensitive to outliers. Additionally, we selected the clustering parameters based on empirical observation. In contrast, in this paper we tested several dimensionality reduction methods to see which ones yielded the best projection for clustering. From all the methods we tested, sphPCA and Isomap (both non-linear) gave us better results. Other dimensionality reduction methods may also be suited for clustering, and we plan to revisit this in future work. In this paper we employ hierarchical clustering to detect intermediate states in the conformational space. The main contributions of this work are as follows:

1. Our parameter choice is automated and based on the properties of the input data, reducing the dependency on user-defined parameters.

2. The hierarchical clustering allows us to view the data from multiple resolutions, detecting intermediate states at a coarser or finer level, at our choice.

3. The clustering is done in the reduced space, thus avoiding the high computational cost of clustering high-dimensional data. Despite that, our clusters are very well-defined even when measuring their properties in the full structural space. (see Tables 1 and 2.)

At first sight, our results seem to be limited by the number of data points or the choice of landmarks. However, persistent Homology is robust enough so that the results do not depend on these, as explained in [20,21].

Finally, as we pointed out earlier, Isomap generally performs better than PCA, at least on the examples
Table 1 Isomap cluster analysis for Calmodulin, AdK and GroEL. The data is visualized in Fig. 3

\begin{tabular}{llll}
\hline Cluster No. & Size & RMSD (1CTR) & RMSD (1CLL) \\
1 & 10 & $14.71 \pm 0.2$ & $1.95 \pm 0.2$ \\
2 & 5 & $13.89 \pm 0.1$ & $2.69 \pm 0.1$ \\
3 & 22 & $13.43 \pm 0.5$ & $3.82 \pm 0.7$ \\
4 & 19 & $10.23 \pm 1.0$ & $6.88 \pm 0.9$ \\
5 & 5 & $7.97 \pm 0.3$ & $8.43 \pm 0.3$ \\
6 & 47 & $3.86 \pm 1.9$ & $11.99 \pm 1.7$ \\
Cluster No. & Size & RMSD (1AKE) & RMSD (4AKE) \\
1 & 15 & $6.49 \pm 0.2$ & $1.99 \pm 0.2$ \\
2 & 19 & $6.04 \pm 0.2$ & $2.85 \pm 0.3$ \\
3 & 6 & $4.96 \pm 0.3$ & $3.73 \pm 0.1$ \\
4 & 8 & $4.65 \pm 0.5$ & $3.84 \pm 0.2$ \\
5 & 10 & $3.56 \pm 0.2$ & $4.42 \pm 0.2$ \\
6 & 33 & $2.91 \pm 0.6$ & $5.91 \pm 0.6$ \\
Cluster No. & Size & RMSD (1SX4) & RMSD (1SS8) \\
1 & 11 & $11.56 \pm 0.2$ & $2.50 \pm 0.6$ \\
2 & 13 & $11.22 \pm 0.2$ & $3.95 \pm 0.5$ \\
3 & 20 & $10.40 \pm 0.5$ & $5.25 \pm 0.5$ \\
4 & 8 & $8.34 \pm 0.5$ & $6.58 \pm 0.4$ \\
5 & 29 & $5.46 \pm 1.2$ & $8.85 \pm 0.7$ \\
6 & 17 & $2.39 \pm 0.9$ & $11.35 \pm 0.6$ \\
\hline
\end{tabular}

The RMSD is measured by the cluster geometric center with respect to each one of the end points. The clusters numbers are sorted according to their RMSD (in $\AA$ ) with respect to their original endpoints

Table 2 Spherical-PCA cluster analysis for Calmodulin, AdK and GroEL

\begin{tabular}{llll}
\hline Cluster No. & Size & RMSD (1CLL) & RMSD (1CTR) \\
1 & 14 & $14.63 \pm 0.3$ & $2.04 \pm 0.4$ \\
2 & 8 & $13.28 \pm 0.4$ & $4.40 \pm 0.4$ \\
3 & 6 & $11.89 \pm 0.5$ & $5.60 \pm 0.3$ \\
4 & 13 & $9.55 \pm 1.0$ & $7.45 \pm 0.6$ \\
5 & 41 & $2.73 \pm 1.4$ & $12.96 \pm 1.2$ \\
Cluster No. & Size & RMSD (1AKE) & RMSD (4AKE) \\
1 & 26 & $6.28 \pm 0.3$ & $2.41 \pm 0.5$ \\
2 & 47 & $2.56 \pm 1.4$ & $5.55 \pm 1.2$ \\
3 & 5 & $2.36 \pm 0.2$ & $6.00 \pm 0.1$ \\
Cluster No. & Size & RMSD (1SX4) & RMSD (1SS8) \\
1 & 25 & $11.42 \pm 0.3$ & $3.17 \pm 0.8$ \\
2 & 14 & $10.20 \pm 0.4$ & $5.31 \pm 0.5$ \\
3 & 39 & $3.74 \pm 1.4$ & $10.24 \pm 1.2$ \\
\hline
\end{tabular}

The RMSD is measured similar to Table 1 
presented. One reason is because, a priori, the topology of the original space will be different to that of the PCAreduced space, since this is given by projection, and in general projections will distort the topology. In contrast, Isomap gives an embedding, which preserves topological features on the components on which the embedding is defined.

\section{Methods \\ Conformational search}

Table 3 shows the proteins used in this work. Each conformational pathway was modeled using a Monte-Carlo (MC) based search described below. Due to the size of the proteins, a fully atomic representation of the structure is computationally costly. Therefore, the proteins were represented using their $\mathrm{C}-\alpha$ atoms, and their potential energy was estimated using a $C-\alpha$ based energy function [22]. The search was run for a maximum of 60,000 iterations. This number was determined experimentally. At every iteration a parent protein conformation is chosen from the conformation pool, then a rotatable bond between two $\mathrm{C}-\alpha$ atoms is selected with a probability linearly proportional to the difference between this angle and its counterpart in the goal conformation, which serves as a bias of the search. Similar angles between start and goal conformation are skipped. The selected angle was rotated by a random value between -5 and 5 degrees. The new conformation is considered further only if its energy is below a threshold. The RMSD of the new conformation with respect to the goal, $\mathrm{RMSD}_{\text {new }}$, is calculated and compared to that of parent conformation, $\mathrm{RMSD}_{\text {parent }}$. The new conformation is accepted and added to the conformation pool according to the Metropolis criterion, if either of the following occurs:

1. $\mid$ RMSD $_{\text {new }}|<|$ RMSD $_{\text {parent }} \mid$

2. $\ln r<-\frac{\left|\mathrm{RMSD}_{\text {new }}\right|-\left|\mathrm{RMSD}_{\text {parent }}\right|}{a\left|\mathrm{RMSD}_{\text {new }}\right|}$,

for a scaling factor $a$, and $r$ the probability of the new conformation. The final result is a pathway leading from the start conformation to the goal conformation. We generated 9543 sampling data points

Table 3 Proteins used in this study. The PDB ids of two known structures of each protein are listed

\begin{tabular}{llll}
\hline Protein & Calmodulin I & AdK & GroEL \\
\hline No. Amino acids & 144 & 214 & 524 \\
Structure 1 & 1 CLL & 1AKE & 1 SS8 \\
Structure 2 & 1 CTR & 4 AKE & $1 S \times 4$ \\
RMSD & 14.84 & 7.13 & 12.21 \\
No. Clusters (Isomap) & 6 & 6 & 6 \\
No. Clusters (PCA) & 5 & 3 & 3 \\
\hline
\end{tabular}

for Calmodulin (1CLL $\rightarrow 1 C T R), 7519$ data points for AdK $(1 \mathrm{AKE} \rightarrow 4 \mathrm{AKE})$ and 11,038 data points for GroEL $(1 \mathrm{SS} 8 \rightarrow 1 \mathrm{SX} 4)$.

\section{Data representation and PCA methods}

The data was represented using several dimensionality reduction methods, which we now describe for completeness.

Robust PCA methods: Standard PCA methods are sensitive to the presence of outliers in the data set. Attempts to overcome this sensitivity are robust generalizations of these linear PCA methods, specifically implemented to either remove outliers or diminish the errors produced by outliers. A non-linear robust dimensionality reduction method is spherical PCA (sphPCA), where the data is scaled so that each data vector is unitary and then one applies standard PCA to the new rescaled data to obtain the principal directions. This method reduces the influence of outliers as explained in [23]. In this paper we use sphPCA, which we found was the most efficient for hierarchical clustering.

Isomap: Isomap is a non-linear dimensionality reduction method that uses multi-dimensional scaling (MDS) [24]. The Isomap algorithm estimates the distances of neighbors using geodesics via a weighted graph, constructed using a $K$-nearest neighbor search to connect the data points, thus preserving linearity only in the small neighborhood of each point (tangent space). In this work we used the minimal $\mathrm{K}$ to generate one connected component. Then, a standard algorithm to obtain the shortest path (geodesic) between two vertices in the graph is performed. Finally, this matrix is subject to MDS, extracting reaction coordinates which determine the embedding.

Conformation space homology, algorithms and generators Quantitative analysis of the conformational space can be done using Algebraic Topology methods. This enables us to study the global properties of conformational spaces as well as to detect rigid local properties, as is the number of local minima in a protein conformational space, by using a natural stratification of the space by energy level. This is, for a given energy $e$, we consider the subsets of conformations $X \leq e$ with energy bounded by $e$, then $X \leq e_{1} \subset X^{\leq e_{2}}$, for $e_{1} \leq e_{2}$. The topology of such subsets will change as the parameter $e$ increases and thus, local minima can be detected. In general, even when the space is given in closed form (by equations), its topology is difficult to determine. In our approach, the spaces are sets of data points generated by the sampling algorithm and thus we have computational restrictions as well. However, we will see that the lower dimensional homology can be determined experimentally. 
Let us describe the topological tools used in our previous work [18]. Suppose $X$ is the (continuous) set of low-energy conformations of a protein, projected to some lower-dimensional metric space. We equip $X$ with the natural topology it inherits from the ambient space. We let $H_{k}(X)$ denote the simplicial homology (with coefficients in a fixed field [25]). We will denote by $b_{k}$ the $k$-th Betti number of $X$, i.e. the dimension of $H_{k}(X)$ as a vector space. The 0 -th Betti number counts the number of connected components or pieces of the space, since any two points are homologous if and only if they are a boundary of a 1-chain. In this paper $X$ will be in general approximated by a discrete set of sample points $Z \subset X$, which is obtained from the conformational search algorithm. We extract information of $X$ from $Z$, using Persistent Homology [26] to estimate the topology of $X$ through algorithms applied to the approximation $Z$. Persistent homology has been successful in detecting topological features of data sets, see for instance [27]. Computational algorithms to obtain persistent homology are described in [20]. In this paper we not only estimate the Betti numbers of $X$, we are interested in finding geometric generators for the homology from the original set. The tools used in the paper are well-suited for data sets. Given a real number $r>0$, we let $C_{*}(Z, r)$ be the simplicial complex whose set of vertices is the set $Z$ itself. We declare the $k$-simplexes as the sets $\left\{x_{0}, \ldots, x_{k}\right\} \subset Z$ if the distance $d\left(x_{i}, x_{j}\right) \leq r$ for all $i \neq j$. The boundary is composed by the maps forgetting one of the vertices. The value of $r$ that we should use to detect the actual topological information of the space $Z$ is initially unknown. The parameter $r$ defines a stream of complexes: for each $r_{1}<r_{2}$ we have $C_{*}\left(Z, r_{1}\right) \hookrightarrow C_{*}\left(Z, r_{2}\right)$, and thus we get natural maps $H_{k}\left(C_{*}\left(Z, r_{1}\right)\right) \rightarrow H_{k}\left(C_{*}\left(Z, r_{2}\right)\right)$ for all $k$. This yields a sequence of vector spaces $H_{k}\left(C_{*}(Z, r)\right)$, and their dimensions $b_{k}(r)$ yield bar codes associated to $Z$, one for each $k$, which encode the evolution of generators of each cohomology (and thus $b_{k}$ ) as $r$ increases (See Fig. 1). These barcodes are formally a set of intervals of the real line, bounded below. A long line in the $k$-th barcode means that there is a $k$-cycle that persists as $r$ increases, and thus it detects an actual generator of the homology of the original space $X$. All small bars are not persistent and are considered noise. We will assume that $r$ will eventually be large enough, say $r_{\max }$, so that $Z$ is covered by balls of radius $r_{\max }$, and thus the complex collapses. We are interested in tracing back the generator for each long bar.

\section{Lazy witness streams and landmarks}

The actual algorithm for computing the bar-codes is a modification of the description above, using landmark points and lazy witness streams. [21]. We specify a set $Z_{0}$ of landmarks in $Z$. $Z_{0}$ is selected according to a sequential mini-max scheme. The first landmark is picked randomly in $Z$. Inductively, if $Z_{0}(i-1)$ is the set of the first $i-1$ landmarks, then we let the $i$-th landmark point to be the point of $Z$ which maximizes the Euclidean distance $d\left(z, Z_{0}(i-1)\right)$ between the point $z$ and the set $Z_{0}(i-1)$. This scheme provides better coverage of the point cloud than a random selection of the landmark points [28]. Given $z \in Z$, we consider the set of all distances $\{d(z, y), y \in$ $\left.Z_{0}\right\}$ from $z$ to $y \in Z_{0}$, and we order them. We denote by $d_{k}(z)$ the $k$-th term, that is the distance from $z$ to its $(k+1)$-closest landmark point. The witness stream complex $W_{k}\left(Z, Z_{0}, t\right)$ has vertices $(k=0) Z_{0}$ and for $k>0$, a $k$-simplex $\left\{z_{0}, \ldots, z_{k}\right\}$ is in $W_{k}\left(Z, Z_{0}, t\right)$ if all of its faces (subsets of cardinality $k$ ) are in $W_{k-1}\left(Z, Z_{0}, t\right)$ and if there is a witness $z \in Z$ (which could be in $Z_{0}$ ) for which $\max \left\{d\left(y, z_{i}\right), i=0, \ldots, k\right\} \leq t+d_{k}(z)$. This clearly defines a stream depending on $t$, since for $t_{1}<t_{2}$, $W_{*}\left(Z, Z_{0}, t_{1}\right) \rightarrow W_{*}\left(Z, Z_{0}, t_{2}\right)$ is an inclusion. Computationally, a witness stream is still quite expensive and thus other modifications are used to estimate Betti numbers. For an integer parameter $v \geq 0$, the lazy witness complex $L W_{*}^{v}\left(Z, Z_{0}, t\right)$ is the stream defined as follows. For $z \in Z$, let $d(z)$ denote the distance from $z$ to the $v$-closest point in $Z_{0}$, just as we did before. Now, define $L W_{0}^{v}\left(Z, Z_{0}, t\right)$ as the set $Z_{0}$. An edge $\left\{z_{0}, z_{1}\right\}$ is in $L W_{1}^{v}\left(Z, Z_{0}, t\right)$ only if there is a witness $y \in Z$ such that $\max \left\{d\left(y, z_{0}\right), d\left(y, z_{1}\right)\right\} \leq t+d(y)$.

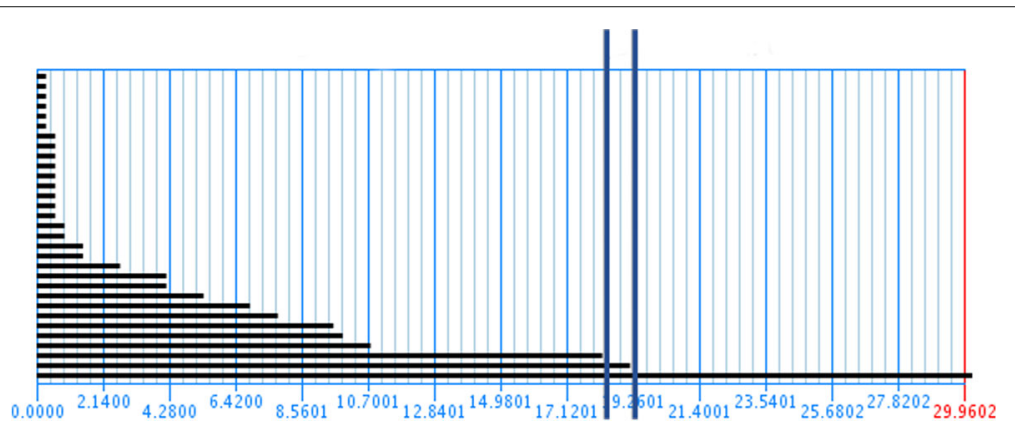

Fig. 1 An example of a barcode diagram. The point where three clusters merge into two, and two merge into one are marked by vertical bars 
The $k>1$ simplex $\left\{z_{0}, \ldots, z_{k}\right\}$ is in $L W_{k}^{v}\left(Z, Z_{0}, t\right)$ if all of its edges are. The lazy witness complex $L W_{*}^{v}\left(Z, Z_{0}, t\right)$ is a flag complex, that is, it is entirely determined by its 1 dimensional skeleton (graph). Note that this modification does not affect the estimation of connected components, but it does depend on $v$. We use either $v=0,1$, however to detect an actual generator of of 0 -th homology (long bar) we use $v=0$. Once a long bar is identified, we find all of the points corresponding to the component using a union-find algorithm, compatible with the Javaplex internal data structures. We then evaluate the cluster that corresponds to these points.

\section{Hierarchical clustering}

To elucidate the topology of the conformational space and to detect intermediate structures we estimated the location of highly populated clusters based on the intervals obtained by the barcodes. The input is the lowerdimensional of the coordinates of a conformational trajectory. We first set out to determine the appropriate number of landmarks for every sample. The choice of landmarks is important to provide sufficient coverage of the conformational space on one hand, and to avoid over-fitting on the other hand. For each sample we ran Javaplex successively with 10, 20,30, etc. landmarks, and measured the variation in $R$, the maximum distance of a point from a landmark. We stop when the difference in $R$ between two consecutive runs is less than $5 \%$. This means that adding more landmarks does not affect the coverage significantly. To account for the randomness in the selection of landmarks, we averaged the resulting number of landmarks over 5 runs and used the average +2 standard deviations. For all the examples in this paper approximately 100 landmarks proved sufficient. Some of these landmarks were outliers and were removed during the clustering. Note that for the method used in this paper, we always use the same set of landmarks in all the JavaPlex runs of the same data set, for consistency.

We then determined the number of clusters systematically by running Javaplex, using the number of landmarks as determined above. The "natural" number of clusters is hard to determine in the general case, but the following heuristic turned out to work well. We successively ran javaplex on the set of coordinates and set the radius $r$ to generate $i$ bars in each run, where $i=\{1,2, \ldots 20\}$. The number of clusters was increased by 1 whenever the difference between two consecutive bars in the barcode plot was more than 0.1 in the barcode plot when $b_{0}$ is calculated. We used the same set of landmarks at each run to make sure the topology of the conformational space and the computational setup is the same each time. To determine the hierarchy between the clusters we checked which cluster split by testing which two sets of landmarks constituting two clusters in the $(i+1)^{s t}$ run were a proper subset of a cluster in the $i^{\text {th }}$ run. This hierarchy between clusters can be displayed using a dendrogram which traces back the relationship between clusters generated by consecutive runs. The clusters are then traced back to the original conformations from the full coordinate space.

\section{Results and discussion}

For each selected protein, the $\mathrm{C}-\alpha$ based representation of the start and target points are used to obtain the conformations pathways that link the two end points by using the MC-based method described in the "Methods" section above. The number of amino acids of each protein is 144 for Calmodulin, 214 for AdK and 524 for GroEL (See Table 3). The dimensionality of the conformational space, representing each conformation by a $3 \times N$ vector with the $x, y, z$ coordinates of each $C$ - $\alpha$, is therefore 432 for Calmodulin, 642 for AdK and 1572 for GroEL. However, the "true" underlying dimensionality for protein structures is much smaller than the number of atomic coordinates requires to represent their structures, due to mutual constraints and interactions between different parts of the proteins. One can see this by computing the variance of the data in the reduced representation. When running sphPCA on the conformational spaces of all the proteins, the first three dimensions account for $90 \%$ or more of the variance in the data. For Isomap, three dimensions explain $>99 \%$ of the variance in the data. The first three coordinates are therefore used in all cases for comparison purposes.

\section{Cluster analysis}

The cluster numbers below are assigned in increasing order of RMSD with respect to one of the endpoints. Table 3 shows the number of clusters detected for each test case. Below, we detail the results for each one of the tested systems.

Calmodulin: The Isomap embedding produced six clusters based on the selected landmarks. One hundred eight landmarks were retained, and the rest discarded as outliers. Table 1 shows each cluster's RMSD with respect to each of the two endpoints, 1CLL and 1CTR. As seen, the clusters span the conformational space between the two end points and the clusters are compact (with small standard deviation of RMSD around the geometric average). The clusters are numbered from 1 to 6 in reverse order of their RMSD with respect to 1CLL (see Table 1). Even though the clusters were numbered according to their RMSD from 1CLL, they are also sorted perfectly with respect to their RMSD from 1CTR. This shows that the clusters span the conformational space between the two endpoints. The distribution of the RMSDs of the cluster centers is not completely uniform, which is probably due to the sampling method which biases the search towards 
the goal structure. Obtaining more uniform sampling is the subject of current work. Figure 2a-f shows the six cluster representative structures, sorted by their RMSD from 1CLL.

Figure 3a illustrates the hierarchy of the clusters for both Isomap and Spherical PCA. Note that the height of the bars is arbitrary (the hierarchy is not, of course). For example - clusters 2 and 3 split from each other at the lowest level, and so are clusters 5 and 6 . However, clusters 2 and 3 are much more similar to one another than clusters 5 and 6, as can be seen both visually in Fig. 2 and from their RMSD to the two end points in Table 1. The average RMSD of the entire set of landmarks is $9.75 \AA$ from 1CLL and 6.15 from 1CTR. As seen in Table 3, the RMSD of the two endpoints is $14.84 \AA$. Figure 4 a shows the Isomap projected landmarks where each resulting cluster is depicted in a different color

The sphPCA analysis resulted in five clusters. The hierarchical relationship and RMSD analysis can be shown in Fig. 3a and on Table 2. As before, the cluster numbers were assigned with respect to the RMSD from 1CLL. The PCA clusters are generally less compact and cover less of the conformational space than in the Isomap case. It can be seen especially when examining Table 2 . The distribution of the clusters is less uniform in the case of PCA, missing parts of the conformational space.

Adenylate Kinase (AdK): Six clusters were detected for AdK, using Isomap. Ninety-one landmarks were retained. The hierarchical relationship and the RMSD analysis of the clusters are shown in Table 1 and Fig. 3b. The cluster numbering was assigned according to the RMSD from 1AKE. Figure 2g-l shows the six cluster representative structures, sorted by their RMSD from 1AKE. Figures 4c shows the Isomap projected landmarks where each cluster is depicted in a different color. As before Table 1 show that the clusters span the entire conformational space even when the RMSD is measured with respect to the other endpoint, 4AKE. The spherical PCA analysis resulted in three clusters. The hierarchical relationship and RMSD analysis can be shown in Fig. 3b and on Table 2. As before, the cluster numbers were assigned with respect to the RMSD from 1AKE. As is the case with Calmodulin, The PCA clusters are generally less compact and cover less of the conformational space than in the Isomap case. Only three clusters were detected this time. For AdK there are known intermediate structures, and further validation is shown below.

GroEL: The Isomap analysis for GroEL produced six clusters containing 98 of the landmarks. The hierarchical relationship and the RMSD analysis of the clusters are shown in Fig. 3c as well as in Table 1. The cluster numbering was assigned according to the RMSD with respect to 1SX4 in descending order. As before, It can be seen in Table 1, that the clusters span the entire conformational space even with respect to the other endpoint, 1SS8. Figure $2 \mathrm{~m}-\mathrm{r}$ shows the six cluster representative structures, sorted by their RMSD from 1SX4. Figure 4e shows the Isomap-projected landmarks where each cluster is depicted in a different color. The generated clusters are less compact than the clusters generated above for Calmodulin and AdK. However, this can be expected since GroEL is a big protein and its conformational transition seems to be more complex than the other two examples.

\section{Validation against known intermediates}

Experimental validation is often difficult to obtain due to lack of experimental knowledge about intermediate structures. However, AdK has several known mutants and intermediate structures [29]. We focused on the following known intermediates: chains $\mathrm{A}, \mathrm{B}$, and $\mathrm{C}$ of the hetero-trimer Adenylate Kinase from Aquifex Aeolicus (PDB code 2RH5), which are conformational change intermediates of the ligand free AdK [30], 1E4Y, which is an

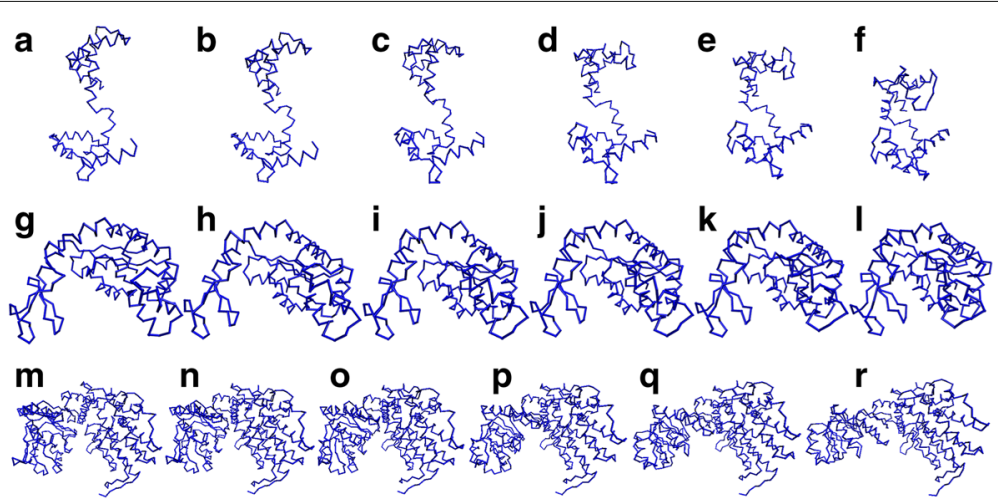

Fig. 2 Representatives of the six cluster centers generated by Isomap for the first case of (a-f) Calmodulin (1CLL $\rightarrow 1 C T R)$. The centers are sorted according to their RMSD from 1CTR. (g-I) AdK. The clusters are sorted according to their RMSD from 1AKE. (m-r) GroEL. The clusters are sorted according to their RMSD from 1SX4 


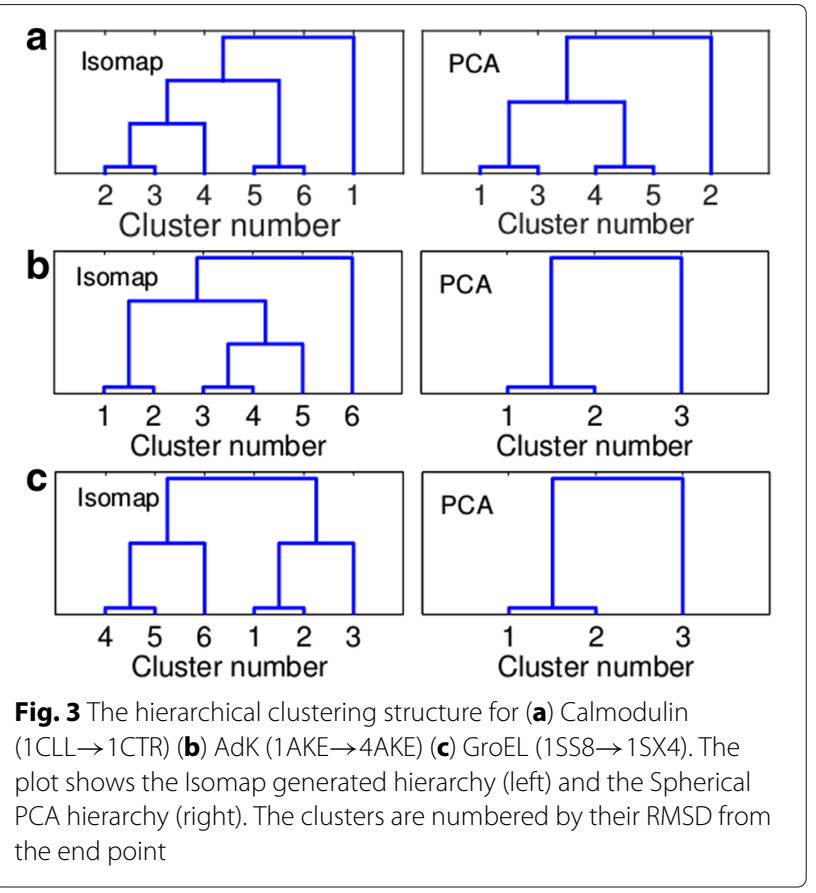

PCA hierarchy (right). The clusters are numbered by their RMSD from
the end point
AdK mutant having 99\% sequence identity with 4AKE and $1 \mathrm{AKE}$ and is a closed form of AdK binding with AP5A, a form of AdK from Bos Taurus (PDB code 1AK2), and a mutant ligated with an ATP analog (PDB code 1DVR). These intermediates have been used successfully to validate conformational pathways for AdK [2, 8, 14, 31]. For each path, we recorded the closest conformation to any of our intermediates. The results are shown in Table 4 . For each intermediate, the table shows the average RMSD from the closest cluster. Our results for Isomap are in good agreement with previous work [29], as well as our earlier studies [14, 32], which predicted 2RH5A-C to be close to the open conformation and 1E4Y to be closest to the closed conformation. Other structures are closer to intermediate conformations. Both Isomap and Spherical PCA were able to find intermediate structures close to 5 intermediates (within about $3 \AA$ or less). However, since Spherical PCA only produced three clusters, it is hard to tell whether the cluster centers span much of the conformational space.

\section{Comparison to K-means clustering}

In order to validate our clustering algorithm, we compared our results to others generated by the K-Means algorithm

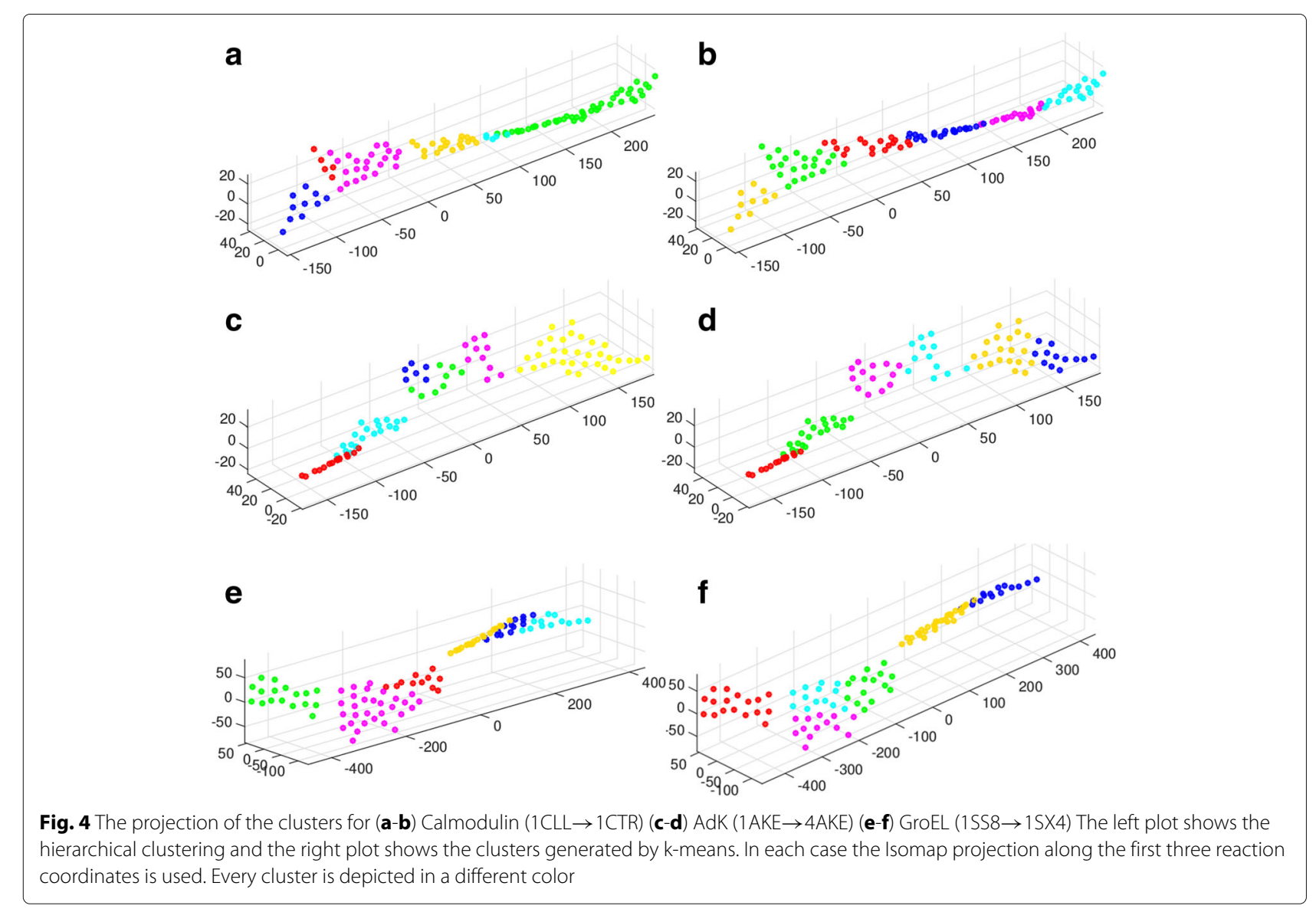


Table 4 Comparison of clusters of AdK to known intermediates

\begin{tabular}{|c|c|c|}
\hline PDB & Isomap closest clust. (RMSD) & PCA closest clust. (RMSD) \\
\hline $1 \mathrm{E} 4 \mathrm{Y}$ & Clust 1 (1.7Å) & Clust $1(2.1 \AA \AA)$ \\
\hline 1 AK2 & Clust $2(3.5 \AA)$ & Clust $1(4.1 \AA \AA)$ \\
\hline 1DVR & Clust $2(2.6 \AA ̊)$ & Clust $1(2.8 \AA \AA)$ \\
\hline $2 \mathrm{RH} 5 \mathrm{~A}$ & Clust $6(2.2 \AA ̊)$ & Clust $3(2.5 \AA)$ \\
\hline 2RH5B & Clust $6(2.3 \AA)$ & Clust $3(2.4 \AA \AA)$ \\
\hline $2 \mathrm{RH} 5 \mathrm{C}$ & Clust $6(3.0 \AA ̊)$ & Clust $3(3.1 \AA \AA)$ \\
\hline
\end{tabular}

For every known intermediate, the RMSD to the closest cluster is shown. The cluster numbers are as in Fig. 2

[33]. K-means is a standard and well-known clustering method, and it is simple to implement. We used the Matlab K-means implementation. For the sake of comparison with our method, we generated six clusters for each one of the Isomap embeddings, which is the same number of clusters produced for each of our examples. As expected, K-means tends to produce roughly equidistant, similar sized clusters, so the results tend to be different than our connected components, which may vary significantly in size and distribution around the center. The K-means clustering of the Isomap data for CaM, AdK and GroEL is shown in Fig. 4b, d, f, respectively. The hierarchical clustering for the Isomap data for CaM, AdK and GroEL is shown in Fig. 4a, c, e, respectively. We placed the two clustering methods next to one another for visual comparison. It is difficult to estimate which structures represent "true" intermediates, especially due to the scarcity of experimental information and the coarse-grained nature of this search. However, the main advantage of our method is that unlike K-means we can easily detect outliers and we can more easily determine the number of clusters. Additionally, the similar sizes and symmetric cluster shapes produced by K-means may produce a bias against the topology and shape of the conformational space.

\section{Conclusions}

Many proteins undergo large-scale conformational changes as part of their function. Characterizing the conformational space of proteins is crucial for understanding their function and dynamics. Finding intermediate conformations which may correspond to local minima is important but highly challenging due to these conformations being transient and the lack of experimental data about intermediate states. We present a persistent homology and dimensionality reduction based hierarchical method to detect clusters of intermediate structures in the conformational spaces of proteins undergoing large-scale changes. The method is able to produce compact clusters that span the conformational spaces of the sampled proteins, and the hierarchical clustering allows us to obtain a multi-scale view. We use a projection to a low-dimensional subspace that preserved the variance in the data. This projection is the input to the persistenthomology based hierarchical clustering, from which intermediate structures are extracted. We tested two non-linear dimensionality reduction methods - Isomap and sphPCA. We find that in general Isomap provides more compact and robust clusters. Future work includes energetic filtering that will allow us to detect high-energy barriers and low-energy folding pathways and domain motions. We also plan to obtain a comprehensive characterization the conformational landscapes of smaller peptides using trajectories produced by all-atom MD simulations.

\section{Acknowledgements}

We thank Henry Adams for all of his help regarding JavaPlex. We thank Tinashe Chagwadera for his help in the initial parts of the project.

\section{Funding}

The research is funded in part by NSF grants No. CCF-1116060 and CCF-1421871 (NH) and by the UMass Boston Healey grant (NH and EG). Publication costs were covered by NSF grant No. CCF-1421871 (NH).

\section{Availability of data and materials}

The code, datasets used and/or analyzed during the current study are available from the corresponding author on reasonable request.

\section{About this supplement}

This article has been published as part of BMC Bioinformatics Volume 18 Supplement 15, 2017: Selected articles from the 6th IEEE International Conference on Computational Advances in Bio and Medical Sciences (ICCABS): bioinformatics. The full contents of the supplement are available online at https://bmcbioinformatics.biomedcentral.com/articles/ supplements/volume-18-supplement-15.

\section{Authors' contributions}

$\mathrm{NH}$ and DL wrote parts of the code and performed the experiments. EG wrote parts of the code and provided the mathematical background. All three authors analyzed the results and participated in writing the manuscript. All authors have read and approved the final manuscript.

\section{Ethics approval and consent to participate}

Not Applicable.

\section{Consent for publication}

Not Applicable.

\section{Competing interests}

The authors declare that they have no competing interests.

\section{Author details}

${ }^{1}$ Department of Computer Science, University of Massachusetts Boston, 100 Morrissey Blvd., 02125 Boston, MA, USA. ${ }^{2}$ Department of Mathematics, University of Massachusetts Boston, 100 Morrissey Blvd., 02125 Boston, MA, USA.

Published: 6 December 2017

\section{References}

1. Miyashita O, Wolynes PG, Onucic JN. Simple energy landscape model for the kinetics of functional transitions in proteins. J Phys Chem B. 2005;109(5):1959-69.

2. Haspel N, Moll M, Baker M, Chiu W, Kavraki LE. Tracing conformational changes in proteins. BMC Struct Biol. 2010;Suppl1:1.

3. Bryngelson JD, Onuchic JN, Socci ND, Wolynes PG. Funnels, pathways, and the energy landscape of protein folding: a synthesis. Proteins. 1995;21:167-95. 
4. Case DA, Cheatham T, Darden T, Gohlke H, Luo R, Jr KMM, Onufriev A, Simmerling C, Wang B, Woods R. The amber biomolecular simulation programs. J Computat Chem. 2005;26:1668-88.

5. Kirkpatrick S, JrCDG, Vecchi MP. Optimization by simulated annealing. Science. 1983;220:671-80

6. Raveh B, Enosh A, Furman-Schueler O, Halperin D. Rapid sampling of molecular motions with prior information constraints. Plos Comp Biol. 2009;5(2):1000295.

7. Shehu A, Olson B. Guiding the search for native-like protein conformations with an ab-initio tree-based exploration. Int J Robot Res. 2010;29(8):1106-27.

8. Al-Bluwi I, Vaisset M, Siméon T, Cortés J. Modeling protein conformational transitions by a combination of coarse-grained normal mode analysis and robotics-inspired methods. BMC Struct Biol. 2013;13(Suppl 1):2.

9. Zheng W, Brooks B. Identification of dynamical correlations within the myosin motor domain by the normal mode analysis of an elastic network model. J Mol Biol. 2005;346(3):745-59.

10. Yang $L$, Song $G$, Jernigan RL. Protein elastic network models and the ranges of cooperativity. Proc Natl Acad Sci. 2009;106(30):12347-52.

11. Schroeder G, Brunger AT, Levitt M. Combining efficient conformational sampling with a deformable elastic network model facilitates structure refinement at low resolution. Structure. 2007;15:1630-41.

12. Frappier V, Chartier M, Najmanovich RJ. Encom server: exploring protein conformational space and the effect of mutations on protein function and stability. Nucleic Acids Res. 2015;43:395-400. doi:10.1093/nar/gkv343. http://nar.oxfordjournals.org/content/early/2015/04/16/nar.gkv343.full. pdf+html.

13. Weiss DR, Levitt M. Can morphing methods predict intermediate structures? J Mol Biol. 2009;385:665-74.

14. Vetro R, Haspel N, Simovici D. Characterizing intermediate conformations in protein conformational space. In: Lecture Notes in Bioinformatics (LNBI) vol. 7845. Berlin: Springer. 2012. p. 70-80.

15. Chang HW, Bacallado S, Pande VS, Carlsson GE. Persistent topology and metastable state in conformational dynamics. PLoS ONE. 2013;8(4):58699.

16. Parsons $L$, Haque $E$, Liu $H$. Subspace clustering for high dimensional data: A review. SIGKDD Explor NewsI. 2004;6(1):90-105.

17. Chazal F, Guibas LJ, Oudot SY, Skraba P. Persistence-based clustering in riemannian manifolds. J ACM. 2013;60(6):41-14138.

18. Haspel N, González E. Topological properties of the configuration spaces of proteins. In: Proc. 4th Int. Conf. on Bioinformatics and Computational Biology (BiCOB). Winona: International Society for Computers and their Applications (ISCA). 2012. p. 245-50.

19. Haspel N, Luo D, Gonzalez E. Detecting intermediate structures in protein conformational pathways. In: Proc. 5th Int. Conf. on Bioinformatics and Computational Biology (BiCOB). Winona: International Society for Computers and their Applications (ISCA). 2013. p. 47-52.

20. Zomorodian A, Carlsson G. Computing persistent homology. Discrete Comput Geom. 2005;33:249-74.

21. de Silva V, Carlsson G. Topological estimation using witness complexes. In: Symposium on Point-Based Graphics, ETH. Aire-la-Ville: Eurographics Association. 2004. p. 157-66.

22. Yap EH, Fawzi NJ, Head-Gordon T. A coarse-grained alpha-carbon protein model with anisotropic hydrogen-bonding. Proteins Struct Funct Bioinforma. 2008;70:626-38.

23. McCoy M, Tropp JA. Two proposals for robust pca using semidefinite programming. Electronic J Stat. 2011;5:1123-60.

24. Tenenbaum JB, De Silva V, Langford JC. A global geometric framework for nonlinear dimensionality reduction. Science. 2000;290(5500):2319-23.

25. Spanier EH. Algebraic Topology. New York: McGraw-Hill Book Co.; 1966, p. 528.

26. Carlsson G. Topology and data. Bull Amer Math Soc (NS). 2009;46(2): 255-308. doi:10.1090/S0273-0979-09-01249-X.

27. Carlsson G, Ishkhanov T, de Silva V, Zomorodian A. On the local behavior of spaces of natural images. Int J Comput Vis. 2008;76:1-12.

28. Adams H, Tausz A. JavaPlex: A research software package for persistent (co)homology. 2011. Software available at http://appliedtopology.github. io/javaplex. Accessed in 2016.

29. Feng $Y$, Yang L, Kloczkowski A, Jernigan RL. The energy profiles of atomic conformational transition intermediates of adenylate kinase. Proteins. 2009;77(3):551-8.
30. Henzler-Wildman KA, Thai V, Lei M, Ott M, Wolf-Watz M, Fenn T, Pozharski E, Wilson MA, Petsko GA, Karplus M. Intrinsic motions along an enzymatic reaction trajectory. Nature. 2007;450(7171):838-44.

31. Molloy K, Shehu A. Elucidating the ensemble of functionally-relevant transitions in protein systems with a robotics-inspired method. BMC Struct Biol. 2013;13(Suppl 1):8.

32. Luo D, Haspel N. Multi-resolution rigidity-based sampling of protein conformational paths. In: Proc. of ACM-BCB (ACM International Conference on Bioinformatics and Computational Biology). New York: ACM. 2013. p. 787-93.

33. MacQueen J. Some methods for classification and analysis of multivariate data. In: Proceedings of 5th Berkeley Symposium on Mathematical Statistics and Probability. 1967. p. 281-97.

\section{Submit your next manuscript to BioMed Central and we will help you at every step:}

- We accept pre-submission inquiries

- Our selector tool helps you to find the most relevant journal

- We provide round the clock customer support

- Convenient online submission

- Thorough peer review

- Inclusion in PubMed and all major indexing services

- Maximum visibility for your research

Submit your manuscript at www.biomedcentral.com/submit
C Biomed Central 